\title{
Low Dose HREM Imaging of Alloyed Metallic and Oxide Nanoparticles Synthesized by Reactive Mechanical Milling
}

\author{
A. Huerta ${ }^{1}$, R. Gonzalez-Flores ${ }^{1}$, M. Ruiz-Ruiz ${ }^{1}$, L.F. Suárez-Quintero ${ }^{1}$, C. Song $^{2}$, C. F. Kisielowski ${ }^{3}$, H. \\ A. Calderon ${ }^{1}$ \\ 1. Dept. Física, ESFM-IPN, UPALM Ed. 9, Mexico D.F., Mexico \\ 2. NCEM-LBNL, Berkeley CA, USA \\ 3. JCAP-NCEM, Berkeley CA, USA
}

Ferrites have interesting properties and can be used as catalysts in several energy applications. Additionally since they are normally magnetic, nanoparticles made of these materials find other uses e.g. biomedical application [1]. Ferrite nanoparticles are normally synthesized in two steps, first a metallic precursor is obtained by using a colloidal technique and then oxidation and annealing give rise to the desired phase. In the present investigation reactive mechanical milling is used to produce pure and alloyed metallic nanoparticles and in a second stage they are oxidized. Reactive milling is done by promoting a chemical reduction of the corresponding chlorides with the use of $\mathrm{Na}$ thus producing $\mathrm{NaCl}$. $\mathrm{NaCl}$ can be easily dissolved if necessary and it is prevents particle agglomeration. Reactive milling can also render alloyed nanoparticles when a mixture of metallic chlorides is processed. The technique is rather simple but a methodological research is needed in order to control size, shape and chemical composition of the products. In this paper results of synthesis and characterization mainly via electron microscopy are reported. A low dose rate is used for imaging in the electron microscope. The TEAM 0.5 is used for atomically resolved characterization of metallic, alloyed and oxide particles. In all cases the accelerating voltage is kept at $80 \mathrm{keV}$.

Figure 1 shows $\mathrm{X}$ ray diffraction patterns of the powders mixtures after different milling times. The systems under investigation consist of $\mathrm{Co}-\mathrm{Fe}, \mathrm{Co}-\mathrm{Mn}$ and $\mathrm{Co}-\mathrm{Cu}$ in various compositions covering the whole possible range. The metallic alloyed nanoparticles form early and develop completely after a short milling time. The nominal chemical composition is mostly preserved in the as milled powders as EDS shows, an example is given in Fig. 2. The as milled powders are washed in $\mathrm{CH}_{3} \mathrm{OH}$ and supported of Lacey $\mathrm{C}$ films for TEM observation. This mainly consists of an exit wave reconstruction (EWR) by using 40 images at different defoci that renders phase and amplitude images. The phase image can be used to retract chemical composition as well as lattice spacings. Fig. 3 shows an oxidized nanoparticle in the $\mathrm{Cu}-20$ at.\%Fe system after $40 \mathrm{~h}$ of milling and room temperature oxidation, the arrow (Fig. 3d) points at the atomic distribution of the oxide with an ordered arrangement of metallic components and a clear difference in the atomic nature of the image columns (different intensities). Alloyed nanoparticles in the system Co-20 at.\% Fe after 40 and $10 \mathrm{~h}$ of milling are given in Figs. 4 and 5, respectively. The experimental images have been taken in low dose rate conditions (70 and $50 \mathrm{e}^{-} / \AA^{2} \mathrm{~s}$ ) to avoid a strong beam sample interaction and the corresponding destruction of the nanoparticles. In both cases, the metallic alloyed nanoparticles show a rather clear and homogeneous contrast in complete agreement with the $\mathrm{X}$ ray diffraction results i.e. the development of nanoparticles with a solid solution of the added components.

\section{References:}

[1] M. Gonzales and K. M. Krishnan, J. Magn. Magnetic Mats. 311 (2007), p. 59.

[2] The financial support of IPN, CONACYT and ICYT DF are gratefully acknowledged. 
[3] NCEM and DOE are acknowledged for the use of TEAM 0.5. at LBNL-NCEM.

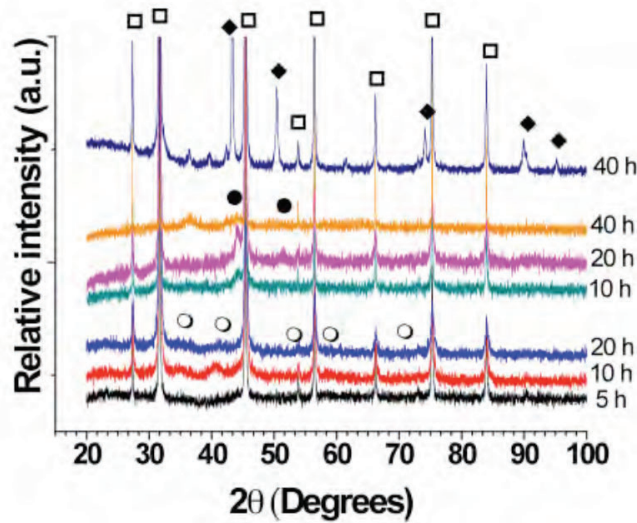

Fig. 1. X-ray diffraction patterns of asmilled $\mathrm{Co}-\mathrm{Fe}$, $\mathrm{Co}-\mathrm{Mn}$ and $\mathrm{Co}-\mathrm{Cu}$ as a function of milling time.

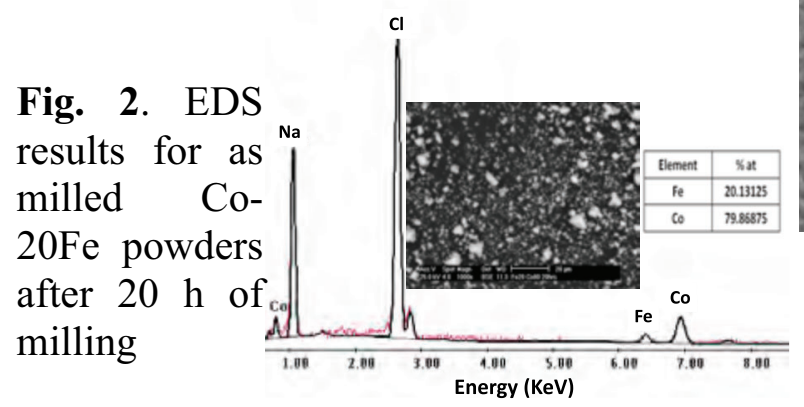

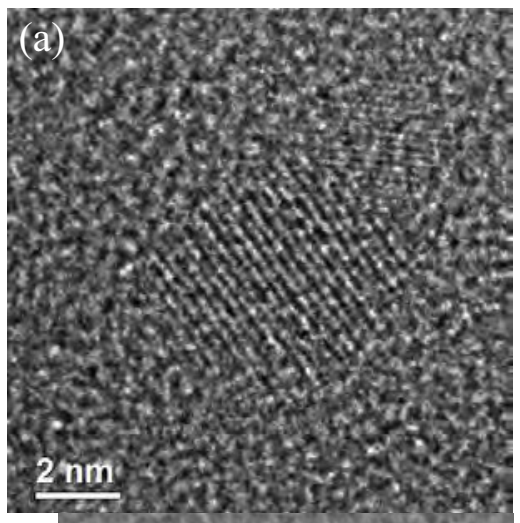

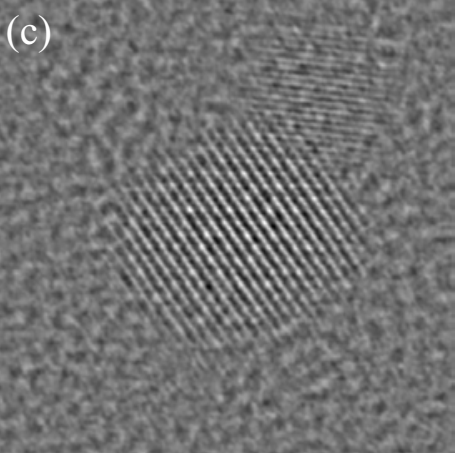

Figure 3. Oxidized nanoparticles from $\mathrm{Fe}-20$ at. $\% \mathrm{Co}$ alloyed powders. (a) Experimental image at a defocus of $220 \mathrm{~nm}$ and a dose rate of $170 \mathrm{e}^{-} / \AA^{2} \mathrm{~s}$, (b) Phase image from EWR procedure, (c-d) Filtered phase images.



Figure 4. Metallic alloyed Co-20 at. \% Fe nanoparticles. (a) Experimental image at $0 \mathrm{~nm}$ of defocus, (b) Phase image (PI) after EWR procedure, (c) background subtraction filtered phase image, (d) colored PI.

Figure 5. Alloyed Co20 at.\% Fe nanoparticles after $10 \mathrm{~h}$ of milling. (a) Experimental image at $220 \mathrm{~nm}$ of defocus and $50 \mathrm{e}^{-} / \AA^{2} \mathrm{~s}, \quad$ (b) Phase image (PI), (c) colored PI.
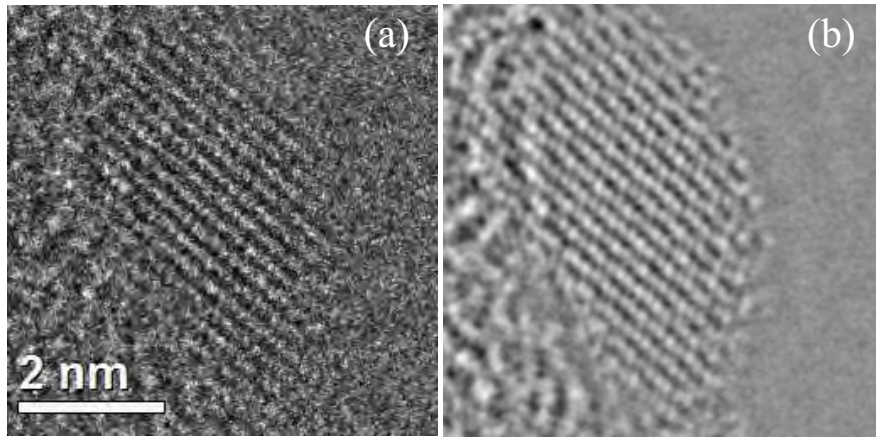